\title{
Prontuários eletrônicos na Atenção Primária: gestão de cadastros duplicados e contribuição para estudos epidemiológicos
}

\author{
Electronic medical records in primary care: management of \\ duplicate records and a contribution to epidemiological studies
}

Luiz Felipe Pinto (https://orcid.org/0000-0002-9888-606X) ${ }^{1}$

Leda Jung dos Santos (https://orcid.org/0000-0002-9554-1621) ${ }^{2}$

${ }^{1}$ Departamento de Medicina em Atenção Primária

à Saúde, Faculdade de Medicina, Universidade Federal do Rio de Janeiro. R. Laura de Araújo $36 / 2^{\circ}$ parte, Cidade Nova. 20211-170 Rio de Janeiro RJ Brasil. felipepinto.rio@ medicina.ufri.br ${ }^{2}$ Curso de Graduação em Medicina, Centro Universitário Serra dos Órgãos. Teresópolis RJ Brasil.

\begin{abstract}
Primary health care electronic medical records were analyzedin Rio de Janeiro for two chronic diseases, namely, hypertension and diabetes, in a population-based study with a crosssectional epidemiological design that considered the Rio de Janeiro population enrolled in Family Health Teams. Calculation of the prevalence rate was stratified by gender and age group, and the condition of the disease was measured by family doctors in their visits using the ICD-10.Except for the last two age groups (75-79 years and 80 years and over), with apparent under-registration of the diagnosed cases, a positive association was found between prevalence rates and age in both genders. The generation of objective and reliable statistical information is fundamental for local management, allowing the evaluation of demographic dynamics and the peculiarities of each territory, and assisting in the planning and monitoring of the quality of Rio de Janeiro people's records registered in each family health unit. Thus, the regular management of duplicate records in the registered user roster is essential to minimize the over-registration of clinical cases reported in the electronic medical records.
\end{abstract}

Key words Electronic medical record, Hypertension, Diabetes mellitus, Primary health care, Brazil
Resumo Analisaram-se os registros eletrônicos da atenção primária em saúde na cidade do Rio de Janeiro para duas doenças crônicas: hipertensão e diabetes, em um estudo de base populacional, com desenho epidemiológico transversal que considerou a população carioca que possuía "Equipes de Saúde da Família". O cálculo da taxa de prevalência foi estratificado por sexo e faixa etária, e a condição da doença foi mensurada pelos médicos de família nas consultas realizadas por estes, computando-se a CID-10. Excetuandose as duas últimas faixas etárias (75 a 79 anos $e$ 80 anos e mais), em que parece haver subregistro dos casos diagnosticados, observou-se uma associação positiva entre as taxas de prevalência e a faixa etária, em ambos os sexos. A geração de informações estatísticas objetivas e com confiabilidade é fundamental para a gestão no nível local, permitindo avaliar a dinâmica demográfica e as particularidades de cada território, e auxiliando no planejamento e monitoramento da qualidade dos registros dos cariocas cadastrados em cada unidade de saúde da família. Para isso, a gestão regular de registros duplicados nas listas de usuários cadastrados é fundamental para minimizar o sobreregistro de casos clínicos apontados nos prontuários eletrônicos.

Palavras-chave Prontuário eletrônico, Hipertensão, Diabetes mellitus, Atenção primária à saúde, Brasil 


\section{Introdução: contextualizando o uso de registros eletrônicos na área da saúde}

A tecnologia de informação em saúde - o hardware, o software e a infraestrutura necessária para coletar, armazenar e trocar informações dos pacientes na prática clínica vem mudando os cuidados em saúde em todo o mundo ${ }^{1}$. Sua implementação envolve um processo complexo com a participação de várias dimensões técnicas, humanas, individuais e organizacionais ${ }^{2}$. A primeira etapa para o uso do software consiste no registro de dados cadastrais e administrativos de todos aqueles que ofertam o cuidado em saúde - as unidades, os profissionais de saúde dos serviços que usam o prontuário. Também devem ser previstos seus aspectos de usabilidade, a qual deve ser maximizada para ganhos em adesão, eficiência e qualidade do registro dos usuários. Estamos nos referindo à questão da necessidade de elaboração prévia e validação pelos gestores, médicos e utilizadores de um dicionário de dados, importante componente que deve ser elaborado quando se constrói um sistema de informação, contendo a descrição do layout das variáveis ${ }^{3}$.

Hendy et al. ${ }^{4}$ destacam o longo período necessário para implementação dos registros eletrônicos em saúde na experiência do Reino Unido. Em 1998, essa previsão era de cerca de 10 anos; contudo, após quatro anos, apenas 3\% dos trusts haviam cumprido a meta estabelecida. As razões apontadas foram a descentralização do financiamento para o nível local e a falta de padronização das tecnologias de informação em saúde. Já Rozenblum et al. ${ }^{5}$ descrevem um projeto canadense de abrangência nacional, iniciado em 2001, que pretendia implementar um sistema único de informação com registros eletrônicos de saúde interoperáveis. Como principal barreira os autores observaram a falta de uma política de $e$-health, a falta de engajamento dos médicos clínicos, a definição de centralizar uma única interoperabilidade nacional, ao invés de se delinear uma abordagem e padrões regionais ${ }^{6}$, a dificuldade de flexibilização do plano de implementação e a não utilização de indicadores clínicos de pagamento por desempenho (pay for performance). Além disso, houve dificuldade de mensurar as metas e os resultados com o investimento reali$\mathrm{zado}^{7}$. Analisando a taxa de implementação dos registros médicos eletrônicos pelos médicos nas províncias do Canadá, Chang \& Gupta ${ }^{8}$ destacam em primeiro lugar a diferença regional no grau de implantação dos mesmos. Apontam que o financiamento neste país não é a principal bar- reira, e que a existência de um médico clínico superutilizador, a liderança regional flexível para implementação e o suporte local são fatores que contribuíram para o sucesso.

A literatura destaca o papel da Dinamarca no êxito para a realização dessa meta. Em um relatório comparativo dos sistemas de saúde da Alemanha, Reino Unido, França, Holanda e Dinamarca, Freudmann \& Studer ${ }^{9}$ destacam que este último país foi o que obteve melhores resultados no uso de registros médicos eletrônicos que reduziram em média 50 minutos por dia o preenchimento de formulários de papel e permitiram atender $10 \%$ a mais pacientes.

Alguns autores ${ }^{10}$ ressaltam que estes registros constituem uma extensa fonte de dados, com baixo custo, que permite comparações longitudinais, estudos de coorte, avaliação da qualidade e ensaios clínicos com grandes tamanhos amostrais $^{11}$, podendo ser utilizados para vigilância em saúde, particularmente das doenças crônicas, como a diabetes, a hipertensão e as cardio-vasculares, que contribuem para grande parte da morbi-mortalidade da populaçãobrasileira ${ }^{12,13}$. Em 2019, o Ministério da Saúde ${ }^{14}$ publicou os resultados da edição mais recente da Pesquisa sobre Vigilância de Fatores de Risco e Proteção para Doenças Crônicas por Inquérito Telefônico - VIGITEL 2018, destacando o município do Rio de Janeiro como a unidade da federação com maior prevalência de hipertensão $(31,2 \%)$ e diabetes $(9,8 \%)$ e acima da média das capitais do País $(24,7 \% \text { e } 7,7 \% \text {, respectivamente })^{15}$. Faz-se relevante destacar que os registros em prontuários são fontes com maior poder e representatividade da saúde da população (morbidade clínica) do que inquéritos populacionais como o VIGITEL, que consideram a morbidade referida.

Neste artigo, consideramos registro eletrônico de saúde a descrição de um conjunto de módulos integrados, interoperáveis e customizáveis, no cuidado diário dos serviços de atenção primária à saúde ${ }^{16}$, contemplando base administrativa, cadastral e base clínica, com ações individuais e atividades em grupo, procedimentos, consultas médicas com registros segundo a Classificação Internacional de Doenças (CID-10), International Classification of Primary Care (ICPC), imunização, visitas domiciliares, resultados laboratoriais e de exames, listas de medicações e de problemas, acessados por todos os profissionais das equipes que compõem os serviços. Estes registros podem ser linkados com outras bases de dados e como a utilização de um código único de identificação como o Cadastro de Pessoa Fí- 
sica (CPF). Além disso, em termos geográficos, o código de endereçamento postal ${ }^{17} \mathrm{e}$ os dados do Censo de um país podem ser utilizados para se obter informações adicionais de determinantes sociais em saúde, servindo inclusive para pesquisas acadêmicas ${ }^{18}$.

O objetivo deste artigo é analisar os prontuários eletrônicos das Equipes de Saúde da Família do município do Rio de Janeiro, apresentando o processo de gestão de cadastros duplicados, o que viabilizou o cálculo das taxas de prevalência de duas das doenças crônicas mais importantes de serem acompanhadas na atenção primária: hipertensão e diabetes.

\section{Métodos}

Estudo de base populacional, com desenho epidemiológico transversal que considerou: (i) as bases administrativas relativas aos dados cadastrais da população carioca que possui "Equipes de Saúde da Família" em Centros Municipais ou Clínicas da Família, (ii) os registros demográficos e clínicos da população de uma determinada área do município do Rio de Janeiro, que iniciou a implementação e o uso de prontuários eletrônicos na APS em meados de 2010. A escolha dessa área ocorreu devido ao fato de ter sido a primeira área da cidade a universalizar o uso dos prontuários eletrônicos, assim como a existência de um Programa de Residência em Medicina de Família e Comunidade ${ }^{19}$.

O município do Rio de Janeiro é o segundo mais populoso do Brasil, o $1.229^{\circ}$ em área geográfica e o $18^{\circ} \mathrm{em}$ densidade populacional. Sua localização estratégica no país e sua importância do ponto de vista histórico, político, econômico e social vêm chamando a atenção dos pesquisadores no mundo, no que se refere à Reforma da Atenção Primária à Saúde, implementada a partir de 2009, conforme destacou a então presidente da WONCA, Professora Amanda Howe ${ }^{20}$. Em 2016, a cidade foi no $21^{\text {st }}$ World Conference of Family Doctors (WONCA ${ }^{21}$ e apresentou sua "Reforma dos Cuidados em Atenção Primária à Saúde"22, em que mais de quatro milhões de cariocas passaram a ter 1.244 equipes próprias de atenção primária à saúde em funcionamento até dezembro/2016 (incluindo-se as Clínicas da Família inauguradas no último trimestre de $2016 \mathrm{e}$ que ainda não continham registros no Cadastro Nacional de Estabelecimentos de Saúde - CNES, em dezembro do mesmo ano), representando em torno de $66 \%$ de cobertura real entre seus moradores - calculada a partir dos registros dos prontuários eletrônicos, após gestão de cadastros duplicados.

Já na perspectiva do Ministério da Saúde, a cidade era, em 2016, o segundo município do Brasil com o maior número de pessoas potencialmente cobertas por Equipes de Saúde da Família nesse período ("cobertura potencial", cujo numerador é calculado a partir do número de ESF $\mathrm{x}$ número médio de 3.450 pessoas cadastradas por ESF). Contudo, posteriormente, a partir de 2017, houve grande redução global do número de equipes cariocas e número de agentes comunitários por equipe e, por consequência, a cobertura potencial de ESF em setembro/2019, caiu para $45,3 \%$ (882 equipes ${ }^{23} \times$ número médio de 3.450 pessoas, dividido por 6.718 .903 habitantes, população estimada para 2019, pelo IBGE).

As unidades que desenvolvem as ações de Atenção Primária em Saúde no município do Rio de Janeiro registradas no Cadastro Nacional de Estabelecimentos de Saúde (CNES) do Ministério da Saúde são os Centros Municipais de Saúde e as Clínicas da Família existentes em dez áreas de planejamento de saúde (AP), os "distritos sanitários" da cidade. Elas são classificadas em unidades 'tipo A', em que existentem apenas Equipes de Saúde da Família (com um médico de família, uma enfermeira, um técnico de enfermagem, seis agentes comunitários de saúde, um agente de vigilância em saúde) e unidades 'tipo B', em que convivem as Equipes de Saúde da Família com profissionais médicos de outras especialidades e outros profissionais de saúde. Todas as Clínicas da Família foram implementadas como unidades 'tipo A' e há Centros Municipais de Saúde, 'tipo A' e 'tipo B'.

Selecionamos as duas doenças crônicas "diabetes" e "hipertensão arterial" para o cálculo da taxa de prevalência ou prevalência pontual, tal como definida por Pereira ${ }^{24}$ (considerando o período de 12 meses - julho/2014 a junho/2015). Estas foram estratificadas por sexo e faixa etária. Inicialmente, estamos interessados em apresentar o processo de gestão de "cadastros duplicados", fundamental para manter a base de dados de identificação única dos usuários limpa e com qualidade para realização de linkages entre outras bases clínicas e dados dos indivíduos. A seguir, pretendemos avaliar a qualidade da codificação dos registros médicos nas consultas realizadas por estes, computando-se a CID-10.

Este estudo foi aprovado pelo Comitê de Ética da Secretaria Municipal de Saúde do Rio de Janeiro no âmbito do Projeto Laboratório de 
Epidemiologia e Estatística da Saúde (LEES) da Atenção Primária e Vigilância em Saúde da cidade do Rio de Janeiro.

\section{A gestão de cadastros duplicados na Secretaria Municipal de Saúde no período de 2013 a 2016: viabilizando o cálculo de indicadores epidemiológicos mais fidedignos}

A linha de base cadastral com dados sociodemográficos foi consolidada no primeiro semestre de 2013, com 171 encontros presenciais descentralizados que envolveram um total de 2.560 agentes comunitários de saúde (ACS), diretores/ gerentes dos Centros Municipais e Clínicas da Família. Essas oficinas de trabalho foram desenvolvidas para qualificar os cadastros definitivos (as chamadas "Fichas A") da população adscrita em todo o município do Rio de Janeiro. Essas fichas continham dados administrativos e sociodemográficos, fundamentais para definir a lista de pessoas de responsabilidade de cada equipe de atenção primária à saúde - que possuía um limite de referência de 3.000 pessoas -, permitindo a construção de uma linha de base para a "gestão da lista de pessoas duplicadas". Essa gestão foi viabilizada a partir do momento em que a Prefeitura do Rio de Janeiro definiu como número único de identificação de cada pessoa o CPF para aquelas com mais de 16 anos e o número da Declaração de Nascidos Vivos (DNV) para quem tivesse menos de 16 anos.

As Oficinas foram realizadas após a impressão e encadernação em papel A3 da lista das variáveis da Ficha A, por microárea e ESF, facilitando com que cada agente comunitário recebesse um caderno e pudesse iniciar o processo de revisão de sua lista de usuários cadastrados e duplicados. Com isso, uma a uma, centenas de milhares de pessoas tiveram seus cadastros atualizados nos prontuários eletrônicos ao longo de seis meses, sob a supervisão das Estações da Rede de Observatórios de Saúde (Rede OTICS-RIO / SMS-RJ).

Após a realização dessas Oficinas, a projeção realizada trouxe evidências de cerca de 340 mil registros de pessoas homônimas com cadastros duplicados, ou seja, $13,7 \%$ das pessoas cadastradas no município (Tabela 1).

Ao longo dos meses que se seguiram, esses cadastros foram sendo revisados e excluídos, ampliando-se, portanto, a possibilidade de acesso e registro de novos moradores das áreas cobertas pelas ESF. Com esse conjunto de oficinas desenvolvidas em 2013, foi possível a criação de uma linha de base que determinou os denominadores a serem utilizados para o cálculo de indicadores clínico-epidemiológicos nos anos seguintes.

Neste estudo, optamos por analisar dois desses indicadores de base populacional, considerando a população-residente de 18 anos ou mais, que totalizava 115.280 habitantes e que eram acompanhados pelas Equipes de Saúde da Família em uma área de planejamento da cidade do Rio de Janeiro.

\section{Resultados e Discussão: o uso de prontuários eletrônicos na Atenção Primária à Saúde e a gestão dos cadastros 'duplicados'}

\section{Prevalência de hipertensão arterial e diabetes}

A análise da situação de saúde de uma determinada área ou território está em permanente construção / reconstrução. Por consequiência, só pode ser compreendida se analisada como decorrente de um processo histórico, em constante transformação. Ferreira ${ }^{25}$ define a situação de saúde como o conhecimento e a interpretação sobre a qualidade da vida da população de um determinado território, historicamente produzido e em permanente processo de transformação. Duas das principais doenças crônicas acompanhadas pelas equipes na atenção primária à saúde referem-se à hipertensão arterial e à diabetes. Do total de pessoas acompanhadas pelas Equipes de Saúde da Família, 4,1\% dos cariocas apresentaram, no período estudado, ambas as morbidades. Quando se observa apenas a hipertensão arterial, a taxa é de 16,7\%, e para diabetes, 9,6\% (Tabela 2).

Nossos achados de base populacional informam taxas superiores no sexo feminino na maior parte das faixas etárias consideradas. Excetuamse as duas últimas faixas etárias ( 75 a 79 anos e 80 anos e mais), nas quais acredita-se haver subregistro dos casos diagnosticados; existindo uma associação positiva entre as taxas de prevalência e a faixa etária, em ambos os sexos. Os idosos desses grupos etários têm mais dificuldade de se deslocar até as unidades de saúde e esta evidência sugere que as equipes de saúde da família poderiam rever e intensificar as necessidades de visitas domiciliares para este subgrupo populacional na área em questão.

A qualidade dos registros que permitiram o cálculo das prevalências de hipertensão e diabetes para a população da zona sul do município do 


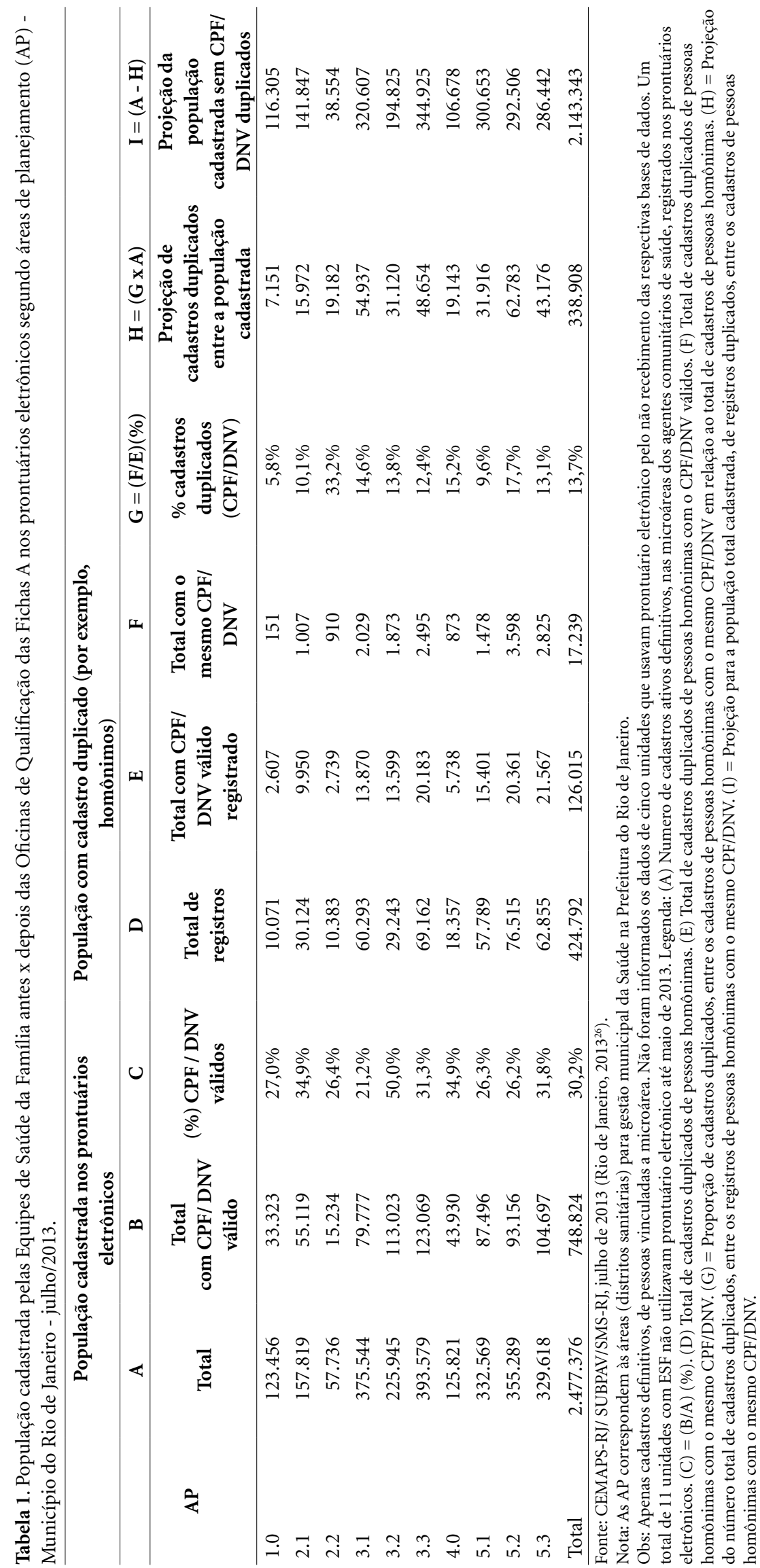


Tabela 2. Prevalência de hipertensão arterial sistêmica e diabetes mellitus diagnosticada pelos médicos de família entre as consultas realizadas por sexo e faixa etária dos usuários. Área selecionada do Município do Rio de Janeiro $(\mathrm{N}=115.280)-2015\left(^{*}\right)$.

\begin{tabular}{|c|c|c|c|c|c|c|c|c|c|}
\hline \multirow{2}{*}{$\begin{array}{c}\text { Faixa } \\
\text { etária (em } \\
\text { anos) }\end{array}$} & \multicolumn{3}{|c|}{$\begin{array}{c}\text { Hipertensão arterial sistêmica } \\
\text { (HAS) }\end{array}$} & \multicolumn{3}{|c|}{ Diabetes } & \multicolumn{3}{|c|}{ (HAS e Diabetes) } \\
\hline & Masculino & Feminino & Total & Masculino & Feminino & Total & Masculino & Feminino & Total \\
\hline De 18 a 24 & $0,5 \%$ & $0,5 \%$ & $0,5 \%$ & - & - & - & $0,0 \%$ & $0,0 \%$ & $0,0 \%$ \\
\hline De 25 a 29 & $1,1 \%$ & $1,2 \%$ & $1,2 \%$ & - & - & - & $0,1 \%$ & $0,0 \%$ & $0,0 \%$ \\
\hline De 30 a 34 & $2,1 \%$ & $3,7 \%$ & $2,9 \%$ & - & - & - & $0,0 \%$ & $0,2 \%$ & $0,1 \%$ \\
\hline De 35 a 39 & $4,2 \%$ & $7,8 \%$ & $6,2 \%$ & - & - & - & $0,4 \%$ & $0,6 \%$ & $0,5 \%$ \\
\hline De 40 a 44 & $8,0 \%$ & $12,9 \%$ & $10,8 \%$ & $2,4 \%$ & $2,7 \%$ & $2,6 \%$ & $1,4 \%$ & $1,7 \%$ & $1,6 \%$ \\
\hline De 45 a 49 & $13,6 \%$ & $21,2 \%$ & $18,0 \%$ & $5,0 \%$ & $4,8 \%$ & $4,9 \%$ & $3,0 \%$ & $3,1 \%$ & $3,1 \%$ \\
\hline De 50 a 54 & $20,7 \%$ & $28,2 \%$ & $25,2 \%$ & $7,0 \%$ & $7,6 \%$ & $7,4 \%$ & $4,9 \%$ & $5,7 \%$ & $5,4 \%$ \\
\hline De 55 a 59 & $28,8 \%$ & $35,9 \%$ & $33,1 \%$ & $10,7 \%$ & $11,5 \%$ & $11,2 \%$ & $7,5 \%$ & $9,3 \%$ & $8,6 \%$ \\
\hline De 60 a 64 & $37,8 \%$ & $42,9 \%$ & $41,0 \%$ & $15,1 \%$ & $14,2 \%$ & $14,6 \%$ & $12,3 \%$ & $12,4 \%$ & $12,3 \%$ \\
\hline De 65 a 69 & $41,2 \%$ & $47,0 \%$ & $44,8 \%$ & $16,8 \%$ & $15,8 \%$ & $16,2 \%$ & $13,6 \%$ & $13,6 \%$ & $13,6 \%$ \\
\hline De 70 a 74 & $42,7 \%$ & $51,2 \%$ & $48, \mathrm{v} 3 \%$ & $16,5 \%$ & $16,9 \%$ & $16,8 \%$ & $13,6 \%$ & $14,6 \%$ & $14,2 \%$ \\
\hline De 75 a 79 & $46,6 \%$ & $49,7 \%$ & $48,7 \%$ & $14,6 \%$ & $17,2 \%$ & $16,4 \%$ & $12,9 \%$ & $15,5 \%$ & $14,7 \%$ \\
\hline 80 ou mais & $39,5 \%$ & $41,2 \%$ & $40,7 \%$ & $13,3 \%$ & $11,7 \%$ & $12,2 \%$ & $11,1 \%$ & $10,4 \%$ & $10,6 \%$ \\
\hline Total & $13,0 \%$ & $19,3 \%$ & $16,7 \%$ & $9,2 \%$ & $9,9 \%$ & $9,6 \%$ & $3,4 \%$ & $4,5 \%$ & $4,1 \%$ \\
\hline
\end{tabular}

Rio de Janeiro foi facilitada pela importância que os médicos residentes que atuam na maior parte das unidades de saúde pesquisadas atribuem aos registros dos dados para a geração de informação para a tomada de decisão.

As estimativas encontradas por sexo e faixa etária permitem seu uso para futuros estudos na área coberta pelas Equipes de Saúde da Família na zona sul da cidade do Rio de Janeiro. Tanto em nossos achados, como no estudo do VIGITEL-2018, as mulheres apresentaram maiores taxas de prevalência. Contudo, para hipertensão arterial, o retrato apresentado pelo Ministério da Saúde para o município do Rio de Janeiro é bastante diferente: $35,3 \%$ vs $16,7 \%$ para a área pesquisada no município do Rio de Janeiro, entre os usuários da APS. Acreditamos que isso ocorra principalmente pelo fato da população de classe média e alta estar presente no estudo brasileiro e, proporcionalmente, menos frequente no estudo com as Equipes de Saúde da Família na cidade do Rio de Janeiro e também a questão da forma de aferição (autoinformada vs registrada em prontuário).

Por outro lado, no período estudado para o município do Rio de Janeiro, a gestão dos cadas- tros duplicados viabilizou o cálculo das prevalências de forma mais adequada do que aquela que tradicionalmente é realizada em sistemas locais de saúde na atenção primária que não realizam a higienização de suas bases de dados e acabam trabalhando com dados agregados (ecológicos). Vários países europeus (Portugal, Espanha, Países Baixos) têm rotinas / algoritmos para gestão dos cadastros duplicados e anualmente atualizam a lista de cada médico / equipe de saúde da família, utilizando inclusive algoritmos informáticos e probabilísticos para a identificação de casos duplicados e junção de registros.

\section{Considerações finais}

Este estudo utilizou pela primeira vez na história da atenção primária à saúde do SUS no município do Rio de Janeiro, registros dos prontuários eletrônicos para o cálculo de prevalências de doenças crônicas com base populacional de uma área de planejamento da cidade, demonstrando a necessidade de monitoramento periódico e gestão regular dos "cadastros de usuários duplicados" na atenção primária à saúde para 
o cálculo de indicadores de saúde mais fidedignos. Os resultados encontrados são pertinentes com aquilo que se esperava na literatura, quanto maior a idade, maior a prevalência de hipertensão e/ou diabetes, com diferenças entre homens e mulheres, demonstrando, portanto, a qualidade dos registros clínicos.

A geração de informações estatísticas objetivas e com confiabilidade é fundamental para a gestão do SUS do micro ao macro, permitindo avaliar a dinâmica demográfica e as particularidades de cada território, e auxiliando no planejamento e no monitoramento dos brasileiros cadastrados em cada unidade de saúde familiar, com a análise contínua da lista de usuários para a retirada de cidadãos com mais de um registro.

Em 2019, o Ministério da Saúde do Brasil definiu algumas inovações para a melhoria da gestão das equipes, uma delas prevê que no novo financiamento federal para a atenção primária ${ }^{27}$, alguns indicadores de monitoramento sejam acompanhados. Para isso, acreditamos ser fundamental a preparação de relatórios gerenciais / algoritmos que possibilitem devolver para cada Equipe de Saúde da Família, sua lista de usuários ativos no ano, lista de usuários cadastrados que não utilizaram os serviços (não utilizadores/ frequentadores) etc. Somente dessa forma será possível o cálculo mais adequado de indicadores clínicos e epidemiológicos, do micro (equipe de saúde da família) ao macro (total do município). Para isso, finalmente, depois de 30 anos de implantação do Sistema Único de Saúde (SUS) e após 25 anos da Estratégia de Saúde da Família, o Governo Federal definiu que o CPF seria o nú- mero único de identificação dos cidadãos brasileiros em dezenas de serviços públicos ${ }^{28}$.

Acreditamos que progressivamente, dada a administração regular pelo Ministério da Economia da base de dados de CPF (ativos, inativos e inválidos), esse processo possa favorecer a redução significativa dos milhões de registros duplicados de usuários das Equipes de Saúde da Família. Afinal, se os resultados (conservadores) da projeção observada para o município do Rio de Janeiro (13\%) forem utilizados para o total do País (que deve ser muito pior, dada a dificuldade dos municípios de pequeno porte em fazer a gestão dos cadastros duplicados), encontraremos cerca de 20 milhões de cadastros duplicados na Estratégia de Saúde da Família, apenas pelo CPF. Se a isso forem somados os duplicados por nome (homônimos; linkage de nome, nome da mãe e data de nascimento) e os cadastrados em mais de uma unidade de saúde ou em mais de um município, outros milhões de registros serão encontrados nessa situação.

O redirecionamento do Ministério da Saúde para a qualificação dos registros individuais é um ponto virtuoso nessa direção, desde que seja acompanhado de um monitoramento contínuo dos registros eletrônicos e apoio das Secretarias Estaduais de Saúde para os pequenos municípios, com menor capacidade de gestão.

Ampliar o acesso é também ampliar a gestão das listas de cidadãos brasileiros, identificando -os univocamente, para que se possa conhecer os verdadeiros denominadores estatísticos para o cálculo dos indicadores pertinentes em cada caso, começando pela cobertura real da Estratégia de Saúde da Família.

\section{Colaboradores}

LF Pinto e LJ Santos participaram igualmente de todas as etapas de elaboração do artigo.

\section{Referências}

1. Canada Health Infoway. The emerging benefits of electronic medical record use in community-based care. Toronto: Health Canada Infoway; 2013.

2. McGinn CA, Grenier S, Duplantie J, Shaw N, Sicotte C, Mathieu L, Leduc Y, Légaré F, Gagnon MP. Comparison of user groups' perspectives of barriers and facilitators to implementing electronic health records: a systematic review. BMC Medicine 2011; 9:46.

3. Pinto LFS. Estratégias de integração e utilização de bancos de dados nacionais para avaliação de políticas de saúde no Brasil [tese]. Rio de Janeiro: Fiocruz; 2006. 
4. Hendy J, Reeves BC, Fulop N, Hutchings A, Masseria C. Challenges to implementing the National Programme for Information Technology (NPfIT): a qualitative study. BMJ 2010; 331(7512):331-336.

5. Rozenblum R, Jang Y, Zimlichman E, Salzberg C, Tamblyn M, Buckeridge D, Forster A, Bates DW, Tamblyn R. A qualitative study of Canada's experience with the implementation of electronic health information technology. CMAJ 2011; 183(5):E281-E288.

6. Webster PC. National standards for electronic health records remain remote. CMAJ 2010; 182(9):888-889.

7. Webster PC. National electronic health records initiative remains muddled, auditors say. CMAJ 2010; 182(9):E383-E384.

8. Chang F, Gupta N. Progress in electronic medical record adoption in Canada. Can Fam Physician 2015; 61(12):1076-1084

9. Freudmann A, Studer S, editors. Future-proofing Western Europe's healthcare - a study of five countries: A report from the Economist Intelligence Unit, 2011. [acessado 2019 Nov 12]. Disponível em: http://www. reforminghealthcare.eu/uploads/Modules/Mediaroom/eiu-eucomed-future-proofing-healthcare-finalv2-web-5-10-11.pdf

10. Schoen C, Osborn R, Doty MM, Squires D, Peugh J, Applebaum S. A survey of primary care physicians in eleven countries, 2009: perspectives on care, costs, and experiences. Health Aff (Millwood) 2009; 28:w11711183.

11. Birtwhistle R, Williamson T. Primary care electronic medical records: a new data source for research in Canada. Can Med Assoc J 2015; 187(4):239-240.

12. Birtwhistle RV. Canadian Primary Care Sentinel Surveillance Network: a developing resource for family medicine and public health. Can Fam Physician 2011; 57(10):1219-1220.

13. World Health Organization (WHO). A Global Brief on Hypertension: Silent Killer, Global Public Health Crisis 2013 [Online]. [acessado 2019 Nov 19]. Disponível em: http://www.thehealthwell.info/node/466541

14. Brasil. Ministério da Saúde (MS). Atividade Física: Vigitel 2016. [acessado 2019 Nov 5]. Disponível em: https://www.endocrino.org.br/minsterio-da-saudedivulga-dados-do-vigitel-2016/

15. Brasil. Ministério da Saúde (MS). VIGITEL Brasil 2018: vigilância de fatores de risco e proteção para doenças crônicas por inquérito telefônico: estimativas sobre frequência e distribuição sociodemográfica de fatores de risco e proteção para doenças crônicas nas capitais dos 26 estados brasileiros e no Distrito Federal em 2018. Brasília: SVS/MS; 2019.

16. Stewart M, Thind A, Terry A, Chevendra V, Marshall J. Implementing and maintaining a researchable database from electronic medical records: a perspective from an academic family medicine department. Health Policy 2009; 5(2):26-39

17. Biro S, Williamson T, Leggett JA, Barber D, Morkem R, Moore K, Belanger P, Mosley B, Janssen I. Utility of linking primary care electronic medical records with Canadian census data to study the determinants of chronic disease: an example based on socioeconomic status and obesity. BMC Medical Informatics and Decision Making 2016; 16:32.
18. Jutte DP, Roos LL, Brownell MD. Administrative record linkage as a tool for public health research. Annu Rev Public Health 2011; 32:91-108.

19. Justino ALA, Oliver LL, Melo TP. Implementation of the Residency Program in Family and Community Medicine of the Rio de Janeiro Municipal Health Department, Brazil. Cien Saude Colet 2016; 21(5):1471-1480.

20. Howe A, Padula AMI, Ribeiro JM, Pinto LF. 450th anniversary of the city of Rio de Janeiro: Primary Health Care Reform. Cien Saude Colet 2016; 21(5):13241326.

21. World Conference of Family Doctors (WONCA). $21^{\text {st }}$ WONCA World Conference of Family Doctors. [acessado 2019 Nov 8]. Disponível em: http://www.wonca2016.com/ingles/index.php

22. Soranz D, Pinto LF, Oliveira GO. Themes and Reform of Primary Health Care (RCAPS) in the city of Rio de Janeiro, Brazil. Cien Saude Colet 2016; 21(5):1327-1338.

23. Brasil. Ministério da Saúde (MS). Secretaria de Atenção Primária à Saúde. Portal eGestor Atenção Básica. (eGESTOR AB). [acessado 2019 Nov 14]. Disponível em: https://egestorab.saude.gov.br/paginas/acessoPublico/relatorios/relHistoricoCoberturaAB.xhtml

24. Pereira MG. Morbidade. In: Pereira MG. Epidemiologia: Teoria e Prática. Rio de Janeiro: Editora Guanabara Koogan; 1995. p. 320-330.

25. Ferreira SM. Curso de Aperfeiçoamento em Gestão de Saúde. In: Programa de Educação à Distância. Sistema de Informação em Saúde. Rio de Janeiro: ENSP; 1998. p. 98.

26. Rio de Janeiro. Prefeitura da Cidade do Rio de Janeiro, Secretaria Municipal de Saúde. Cadernos de Estatísticas e Mapas da Atenção Primária em Saúde do Município do Rio de Janeiro (CEMAPS). Contribuições para a construção de uma linha de base para os cadastros das microáreas da estratégia de Saúde da Família do município do Rio de Janeiro. Rede de Estações-Observatório das Tecnologias de Informação e Comunicação em Serviços de Saúde. Rio de Janeiro: Secretaria Municipal de Saúde; 2013.

27. Brasil. Portaria MS no 2.979, de 12 de novembro de 2019. Institui o Programa Previne Brasil, que estabelece o modelo de financiamento de custeio da atenção primária à saúde no âmbito do SUS. Diário Oficial da União 2019; 13 nov.

28. Brasil. Decreto no 9.795, de 17 de maio de 2019. Diário Oficial da União 2019; 20 maio.

Artigo apresentado em 22/11/2019

Aprovado em 20/12/2019

Versão final apresentada em 22/12/2019 